\title{
Efecto de la temperatura y la acidificación en larvas de Strombus gigas (Mesogastropoda: Strombidae)
}

\author{
José Francisco Chávez Villegas ${ }^{1}$, Martha Enríquez Díaz ${ }^{1}$ \& Dalila Aldana Aranda ${ }^{1}$ \\ Laboratorio de Conservación, Cultivo y Biología de Moluscos, Centro de Investigación y de Estudios Avanzados del \\ Instituto Politécnico Nacional, Unidad Mérida, Carretera Antigua a Progreso, Km. 6, A.P. 73 Cordemex, C.P. 97310, \\ Mérida,YYucatán, México; biol.chavez@gmail.com, marthaenriquez_1999@yahoo.com, daldana@mda.cinvestav.mx
}

$$
\text { Recibido 01-VII-2016. Corregido 14-XI-2016. Aceptado 15-XII-2016. }
$$

\begin{abstract}
Effect of temperature and acidification in Strombus gigas (Mesogastropoda: Strombidae) larvae. The increase in $\mathrm{CO}_{2}$ emissions produces heating and reduced $\mathrm{pH}$ in the oceans, which may have negative effects on many marine organisms. This is particularly important for those with calcified structures such as the molluses and their larval stages. We studied Strombus gigas larvae, a gastropod of commercial importance in the Caribbean Sea, in order to know the effect of water temperature and acidification on their development, growth, mortality and calcification during the larval period. A larval culture with triplicate samples was carried out employing four treatments of temperature and $\mathrm{pH}\left(\mathrm{Control}=28{ }^{\circ} \mathrm{C}-\mathrm{pH} 8.1, \mathrm{~T} 1=28{ }^{\circ} \mathrm{C}-\mathrm{pH} 7.6, \mathrm{~T} 2=\right.$ $31{ }^{\circ} \mathrm{C}-\mathrm{pH} 8.1$ and $\mathrm{T} 3=31{ }^{\circ} \mathrm{C}-\mathrm{pH}$ 7.6) in August 2015. We registered hatching (No. of eggs - No. of larvae hatched) and organs development, while shell growth and mortality ratio were evaluated over time. Shell calcification was studied in 30 days old larvae using EDX and RAMAN analysis. Our results showed that organs development and shell growth were higher at $31^{\circ} \mathrm{C}$ treatments (initial size of $230 \pm 4.12$ to $313.27 \pm 11.34 \mu \mathrm{m}$, and final size from $829.50 \pm 11.33$ to $1054.50 \pm 11.13 \mu \mathrm{m}$; from $\mathrm{T} 1$ to $\mathrm{T} 2$ respectively), and the same pattern was recorded for hatching time $(18 \mathrm{hr})$ and mortality rate $(\sim 57 \%)$. The Calcium proportion $(\% w t)$ was similar between treatments $(34.37 \pm 10.05$ to $37.29 \pm 16.81 \% w t)$. Shell Raman analysis showed aragonite in all experimental treatments, with the highest values in the control (1 $039.54 \pm 780.26$ a.u.). Calcite was detected only in $31{ }^{\circ} \mathrm{C}$ treatments $(174.56 \pm 127.19$ a.u. $)$, while less intensity of aragonite and calcite were registered at $\mathrm{pH}$ 7.6. In conclusion, S. gigas could be adapted to ocean future predictions, however, shell biomineralization processes can be affected. Rev. Biol. Trop. 65 (2): 505-515. Epub 2017 June 01.
\end{abstract}

Key words: development, temperature, acidification, calcification, Strombus gigas.

El incremento en las emisiones de $\mathrm{CO}_{2}$ durante el último siglo, ha producido cambios en los océanos, produciendo aumento de la temperatura y disminución del $\mathrm{pH}$ en ellos (Intergovernmental Panel on Climate Change, 2007). En el caso particular del mar Caribe, Chollett, Mumby, Müller-Karger, y Hu (2012), reportan que la temperatura ha aumentado $1.41{ }^{\circ} \mathrm{C}$ durante el último siglo (de $0.19{ }^{\circ} \mathrm{C}$ a $0.27{ }^{\circ} \mathrm{C}$ por decenio), alcanzando una temperatura media de $28{ }^{\circ} \mathrm{C}$. Por otra parte, Magaña Rueda, Graizbord, Buenfil Friedman y Gómez Mendoza (2009) sugieren que la temperatura en esta región podría ascender hasta los $31{ }^{\circ} \mathrm{C}$ hacia el año 2100.

Modelaciones de acidificación señalan que el $\mathrm{pH}$ de los océanos podría descender de 8.1 (media actual) a 7.6 hacia el año 2100 (Caldeira \& Wickett, 2003; Orr et al., 2005). De acuerdo con Gledhill, Wanninkhof, Millero y Eakin (2008), se calcula que la presión parcial de $\mathrm{CO}_{2}$ $\left(\mathrm{pCO}_{2}\right)$ en el Caribe ha aumentado $+2.2 \mu \mathrm{atm}$. $\mathrm{año}^{-1}$ durante el último siglo, produciendo una disminución de 0.05 unidades de $\mathrm{pH}$.

Cambios en estos parámetros afectan directamente la dinámica de las poblaciones, 
especialmente a organismos calcificadores, los cuales son más vulnerables durante su fase larvaria (Sale et al., 2010).

Roller y Strickle (1989), observaron que al aumentar la temperatura (de 20 a $30{ }^{\circ} \mathrm{C}$ ) se presenta un incremento en las tasas de desarrollo, crecimiento y mortalidad para Thais haemastoma canaliculata, por otra parte, Watson, Southgate, Tyler, y Peck (2009), mencionan que larvas de Saccostrea glomerata expuestas a reducción de $\mathrm{pH}$ (de -0.1 a -0.2 unidades) presentan disminución en sus tasas de crecimiento y desarrollo, así como una esqueletogénesis anormal.

Strombus gigas (Linnaeus, 1758) es un organismo con distribución metapoblacional en el Caribe, presenta un estadio larval, cuya cinética de desarrollo ha sido estudiada en condiciones normales de temperatura $\left(28-29^{\circ} \mathrm{C}\right)$, por lo que no se conoce el efecto que el incremento de temperatura y la disminución del $\mathrm{pH}$ ejercen durante su etapa larval. En este sentido, la finalidad del presente estudio fue evaluar el efecto del incremento de la temperatura y la acidificación en la eclosión, desarrollo, crecimiento, mortalidad y calcificación de la concha durante su fase larvaria.

\section{MATERIALES Y MÉTODOS}

Cultivo larval: Se recolectó un segmento de masa ovígera de $S$. gigas recién desovada en Quintana Roo $\left(20^{\circ} 19^{\prime} 00^{\prime}\right.$ 'N - 87²1'25” W, agosto 2015), la cual fue transportada para su cultivo a la estación Marina del Cinvestav-IPN (Telchac, Yucatán, 2120'28" N - 89¹8'25" W). La incubación de embriones y el cultivo larval se realizaron de agosto a octubre 2015 , empleando la condición $28^{\circ} \mathrm{C}-\mathrm{pH} 8.1$ como control y tres tratamientos de temperatura $\mathrm{y}$
$\mathrm{pH}\left(\mathrm{T} 1=28{ }^{\circ} \mathrm{C}-\mathrm{pH} 7.6, \mathrm{~T} 2=31{ }^{\circ} \mathrm{C}-\mathrm{pH}\right.$ $8.1, \mathrm{~T} 3=31{ }^{\circ} \mathrm{C}-\mathrm{pH}$ 7.6.), por triplicado. Los niveles de temperatura y $\mathrm{pH}$ utilizados se establecieron de acuerdo a las condiciones actuales y las proyecciones hacia 2100 para la región Norte del Caribe Mexicano (Caldeira \& Wickett, 2003; Chollett et al., 2012; Magaña Rueda et al., 2009). La temperatura se controló utilizando calentadores para acuarios. El pH de los tratamientos se reguló mediante burbujeo, suministrando $\mathrm{CO}_{2}$ cada $4 \mathrm{hr}$. Temperatura y $\mathrm{pH}$ se monitorearon con un potenciómetro Oakton Mod. pH 5+.

En cada unidad de cultivo se colocó un segmento de masa ovígera de $15 \mathrm{~cm}$ de longitud ( \pm 106 huevos por $\mathrm{cm}$ lineal). Se registró el tiempo a la eclosión (hr) para cada tratamiento. A partir de la eclosión, las larvas fueron distribuidas manualmente a unidades de cultivo de 5 $\mathrm{L}$ a razón de 160 larvas. $\mathrm{L}^{-1}$.

La alimentación se realizó por las mañanas (frecuencia: $24 \mathrm{hr}$ ), suministrando una mezcla de microalgas (Nannochloropsis oculata, Isochrysis galbana, Chaetoceros mulleri, Pavlovav sp. y Navicula sp.), a una concentración total de 1000 cel. $\mathrm{mL}^{-1}$ por unidad de cultivo (200 cel. $\mathrm{mL}^{-1}$ de cada microalga por ración diaria).

Se realizaron recambios totales cada 5 días empleando agua de mar filtrada mediante filtros de polipropileno $(5 \mu \mathrm{m})$ y esterilizada con UV. Se utilizaron mallas de $180 \mu \mathrm{m}$ para la retención de las larvas.

Eclosión, desarrollo, crecimiento y mortalidad: Se registró el tiempo inicial y final de eclosión para cada tratamiento, posteriormente, se contabilizó el número de huevos no eclosionados por segmento de masa ovígera. El porcentaje de eclosión se calculó de la siguiente manera:

$$
\% \text { de eclosión }=\frac{\mathrm{N} .^{\circ} \text { inicial de huevos }-\mathrm{N} .^{\circ} \text { de huevos sin eclosionar }}{\mathrm{N} .^{\circ} \text { inicial de huevos }} * 100
$$

A fin de evaluar el desarrollo, cada 24 hr usando una pipeta Pasteur se extrajeron 30 larvas por tratamiento, las cuales se observaron (in vivo) a través un microscopio óptico LEICA
ICC50. El desarrollo se observó de acuerdo a Brito-Manzano, Aldana-Aranda, de la CruzLázaro, y Estrada-Botello (2006), los indicadores fueron: número de lóbulos del velum, 
número de espiras de la concha, corazón funcional (larval y adulto), opérculo, branquias (Fig. 1 A) y conducta nado-reptante. El crecimiento ( $\mathrm{N}=30$ larvas por edad/tratamiento) y mortalidad fueron evaluados en larvas de 0,10 , 20 y 30 días. Se midió la longitud sifonal $(\mu \mathrm{m})$ de la concha (Fig. 1 B) utilizando el software Leica LAS Interactive Measurements (resolución 10x). La mortalidad fue calculada a partir de las conchas vacías obtenidas durante los recambios de agua. El porcentaje de mortalidad se obtuvo con la siguiente fórmula:

$$
\% \text { de mortalidad }=\frac{\mathrm{N} .^{\circ} \text { de conchas vacías }}{\mathrm{N} .^{\circ} \text { inicial de larvas }} * 100
$$

Calcificación: Se determinó la proporción en peso de Calcio ( $\% w t)$ para larvas de 30 días ( $\mathrm{N}=3$ larvas por tratamiento), se eligió esta edad dado que la teleoconcha se forma entre los 30 y 35 días (Enriquez-Díaz, Volland, Chavez-Villegas, Aldana-Aranda, \& Gros, 2015). Para la cuantificación se utilizó Microscopia Electrónica de Barrido empleando el Análisis de Espectroscopía de Energía Dispersiva "EDX" (Microscopio PHILIPS ESEM-XL30). La medición se hizo en cuatro zonas de la concha siguiendo la longitud sifonal (Fig. 1B). Además, se utilizó Microscopía RAMAN (Microscopio WITEC ALPHA 300, Láser 488nm), empleando las distancias raman $\left(\mathrm{cm}^{-1}\right)$ propuestas por Nehrke, Poigner, Wilhelms-Dick, Brey, \& Abele (2012) para la identificación y cuantificación de aragonita $\left(206,705\right.$ y $\left.1085 \mathrm{~cm}^{-1}\right)$ y calcita $(282,711$ y $\left.1085 \mathrm{~cm}^{-1}\right)(\mathrm{N}=3-5$ larvas por tratamiento). El efecto de fluorescencia registrado en las muestras se eliminó mediante el software CrystalSleuth-RRUFF Project. La intensidad RAMAN se calculó con el software OriginPro 8 , y se reportó en unidades arbitrarias (a.u.).

Se utilizó el software Infostat versión estudiantil, para calcular media y desviación estándar, así como realizar prueba de normalidad (Shapiro-Wilks), de homocedasticidad (Kolmogorov) y análisis de Kruskal-Wallis ( $\mathrm{P}$ $(\alpha 0.05)$ para eclosión, crecimiento, mortalidad $\mathrm{y}$ calcificación (EDX) entre tratamientos de temperatura y $\mathrm{pH}$.

\section{RESULTADOS}

Eclosión: Se presentó a partir del día cinco de incubación, iniciando en larvas de $31{ }^{\circ} \mathrm{C}$ a partir de las $110 \mathrm{hr}$ posteriores al desove, y las $118 \mathrm{hr}$ para los tratamientos
A

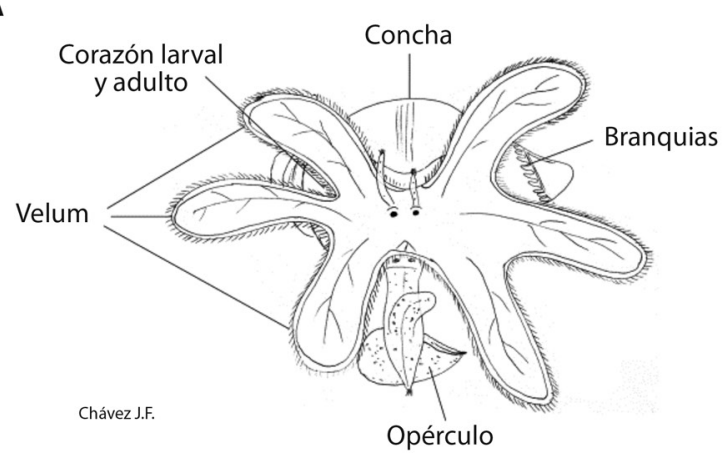

B

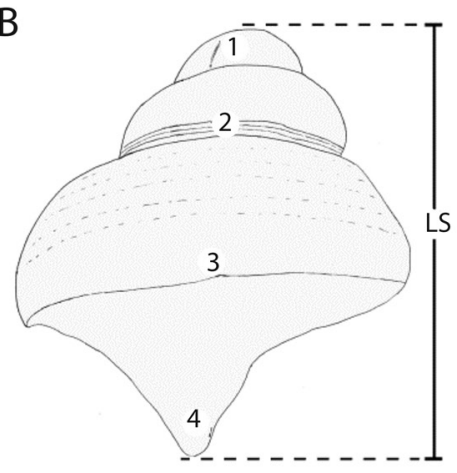

Fig. 1. Larva de Strombus gigas (28 días), donde se muestran: A) Órganos utilizados para evaluar su desarrollo, B) Zonas utilizadas para medir la longitud sifonal (LS) y para la cuantificación de calcio mediante análisis EDX. 1 = ápice en la primera espira, 2 = ornamentaciones de la segunda espira, 3 = cuerpo de la concha fuera de ornamentaciones, $4=$ canal sifonal.

Fig. 1. Original diagrams of S. gigas larvae (28 days old), showing: A) Organs used to evaluate larval development; B) Areas used to measure siphonal length (LS) and to perform Calcium measurements by EDX analysis. $1=$ apex in the first shell whorl, 2 = ornamentations of the second shell whorl, 3 = body shell outside ornamentations, $4=$ siphonal channel. 
de $28{ }^{\circ} \mathrm{C}$. La duración de la eclosión fue de $18 \mathrm{hr}$ para $31{ }^{\circ} \mathrm{C}$ y de $30 \mathrm{hr}$ para $28^{\circ} \mathrm{C}$ (Fig. 2A). En general, se registró $\sim 90 \%$ de eclosión, con excepción del T2 $\left(31^{\circ} \mathrm{C}-\mathrm{pH} 8.1\right)$ donde se obtuvo un $96.67 \pm 1.39 \%$ de larvas eclosionadas (Fig. 2B). No hubo variación significativa en la proporción de larvas eclosionadas entre tratamientos $\left(\mathrm{H}_{\text {(g.l. 3) }}=6.38\right.$, $\left.\mathrm{P}_{(\alpha 0.05)}=0.0943\right)$, pero si en el tiempo de eclo$\operatorname{sión}\left(\mathrm{H}_{(\mathrm{g} .1 .3)}=8.31, \mathrm{P}_{(\alpha 0.05)}<0.0001\right)$.
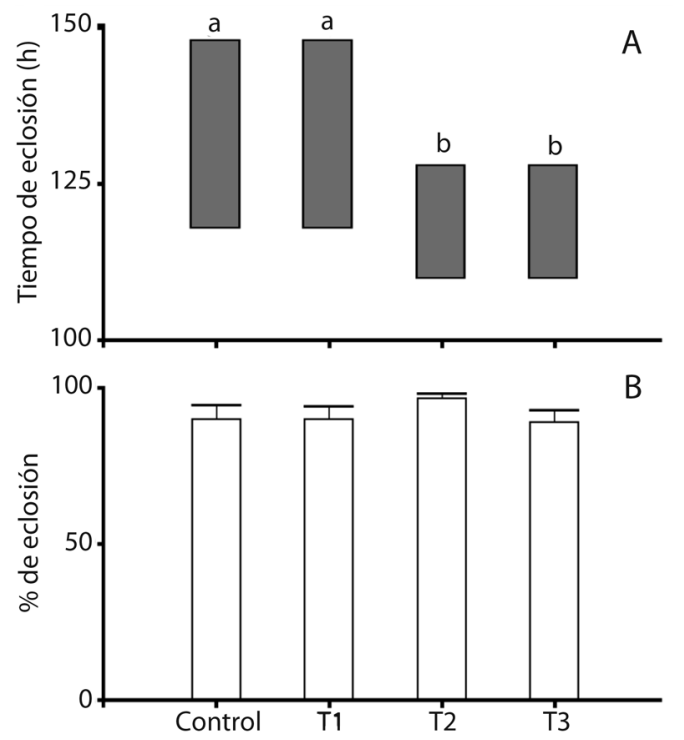

Fig. 2. Observaciones realizadas durante la eclosión (A: rango, B: porcentaje) para larvas de $S$. gigas para los tratamientos de temperatura $\left({ }^{\circ} \mathrm{C}\right)$ y $\mathrm{pH}$. Control $=28$ ${ }^{\circ} \mathrm{C}-\mathrm{pH} 8.1, \mathrm{~T} 1=28{ }^{\circ} \mathrm{C}-\mathrm{pH} 7.6, \mathrm{~T} 2=31{ }^{\circ} \mathrm{C}-\mathrm{pH} 8.1$, $\mathrm{T} 3=31{ }^{\circ} \mathrm{C}-\mathrm{pH}$ 7.6. a-b: grupos generados a partir de la comparación de medias de Kruskal-Wallis ( $\alpha 0.05)$.

Fig. 2. Observations of hatchery process (A: range, B: percentage) of the $S$. gigas larvae for the temperature $\left({ }^{\circ} \mathrm{C}\right)$ and $\mathrm{pH}$ treatments. Control $=28^{\circ} \mathrm{C}-\mathrm{pH} 8.1, \mathrm{~T} 1=28{ }^{\circ} \mathrm{C}$ - $\mathrm{pH} 7.6, \mathrm{~T} 2=31{ }^{\circ} \mathrm{C}-\mathrm{pH} 8.1, \mathrm{~T} 3=31{ }^{\circ} \mathrm{C}-\mathrm{pH}$ 7.6. a-b: groups obtained from average comparisons by KruskalWallis analysis $(\alpha 0.05)$.

Desarrollo larvario: La cinética de desarrollo del velum fue mayor para larvas de 31 ${ }^{\circ} \mathrm{C}-8.1 \mathrm{pH}$ (T2), donde la reducción de lóbulos velares (indicador del inicio de la metamorfosis) comenzó a parir del día 38, mientras que en el control $\left(28^{\circ} \mathrm{C}-\mathrm{pH} 8.1\right)$ se observó a partir del día 41. La concha mostró menor desarrollo en larvas del $\mathrm{T} 1\left(28^{\circ} \mathrm{C}-\mathrm{pH} 7.6\right)$, las cuales no desarrollaron 4.5 espiras, característica observada en el resto de los tratamientos. La elongación del opérculo, la aparición del corazón adulto funcional, el desarrollo de las branquias e inicio de la conducta nado-reptante, se presentó en menor tiempo y mayor proporción en los tratamientos de $31^{\circ} \mathrm{C}$ (Cuadro 1$)$.

Crecimiento: Las larvas presentaron tallas de $230 \pm 4.12 \mu \mathrm{m}\left(28^{\circ} \mathrm{C}-\mathrm{pH} 7.6\right)$ a $313.27 \pm$ $11.34 \mu \mathrm{m}\left(31^{\circ} \mathrm{C}-\mathrm{pH}\right.$ 8.1) durante la eclosión. Se registró un crecimiento similar entre el control y los tratamientos hacia el día 10 (L.S. $=\sim 650 \mu \mathrm{m}$ ), hacia el día 20, larvas de los tratamientos $31^{\circ} \mathrm{C}$ fueron más grandes $\left(28{ }^{\circ} \mathrm{C}=\right.$ $\sim 710 \mu \mathrm{m}$ y $31{ }^{\circ} \mathrm{C}=\sim 820 \mu \mathrm{m}$ ), mismo patrón se registró durante todo el desarrollo, alcanzando las mayores tallas en los tratamientos de 31 ${ }^{\circ} \mathrm{C}(\mathrm{pH} 7.6=1031.50 \pm 11.03 \mu \mathrm{m} ; \mathrm{pH} 8.1=$ $1054.50 \pm 11.13 \mu \mathrm{m}$ ) (Fig. 3). El análisis de Kruskal-Wallis mostró variación significativa en el crecimiento entre tratamientos $\left(\mathrm{H}_{(\mathrm{g} .1 .3)}=\right.$ $\left.26.19, \mathrm{P}_{(\alpha 0.05)}<0.0001\right)$.

Mortalidad: Se registró de $0.59 \pm 0.19 \%$ a $3.01 \pm 0.25 \%$ de mortalidad durante el día 10 (Fig. 4A), incrementando de $1.38 \pm 0.54 \%$ a $5.67 \pm 1.16 \%$ hacia el día 20 (Fig. 4B). Se presentó un pico de mortalidad el día 30 para todos los tratamientos (de 19.13\% a 67.38), mostrando mayores registros en el $\mathrm{T} 3$ con $58.42 \pm 12.03 \%$ (Fig. 4C). El mayor porcentaje de mortalidad acumulada fue en los tratamientos de $31^{\circ} \mathrm{C}(\mathrm{pH} 8.1=65.64 \pm 8.49 \%$ y pH 7.6 $=65.10 \pm 15.49 \%$ ). La mortalidad no mostró variación significativa entre tratamientos $(\mathrm{H}$ $\left.{ }_{(\mathrm{g} .1 .3)}=5.41, \mathrm{P}_{(\alpha 0.05)}=0.1441\right)$, mientras que en el análisis por edad, se observó variación solo para las observaciones realizadas durante el día $20\left(\mathrm{H}_{(\mathrm{g} .1 .3)}=8.74, \mathrm{P}_{(\alpha 0.05)}=0.0324\right)$.

\section{Calcificación:}

EDX: La proporción de Calcio $(\% w t)$ fue similar entre tratamientos $\sim 35 \% w t$, siendo mayor en el control con $37.29 \pm 16.81 \% w t$ 
CUADRO 1

Día de cultivo y avance (\%) en de aparición de órganos (velum, concha, corazón, opérculo y branquias) y conducta nado-reptante en larvas de $S$. gigas para los tratamientos temperatura y $\mathrm{pH}$

TABLE 1

Culture days and advance (\%) of organs development (velum, shell, heart, operculum and gills) and swim-crawling behavior in S. gigas larvae of the temperature and $\mathrm{pH}$ treatments

\begin{tabular}{lccccccccc}
\multirow{2}{*}{ Característica } & Grado de desarrollo & \multicolumn{2}{c}{ Control } & \multicolumn{2}{c}{ T1 } & \multicolumn{2}{c}{ T2 } & \multicolumn{2}{c}{ T3 } \\
& & Día & $\%$ & Día & $\%$ & Día & $\%$ & Día & $\%$ \\
Velum (N de lóbulos) & Un par & 0 & 100.0 & 0 & 100.0 & 0 & 100.0 & 0 & 100.0 \\
& Dos pares & 2 & 100.0 & 2 & 100.0 & 1 & 86.7 & 2 & 100.0 \\
& Tres pares & 6 & 93.6 & 5 & 83.0 & 3 & 76.7 & 4 & 70.0 \\
& Reducción & 41 & 23.3 & 41 & 23.3 & 38 & 10.0 & 40 & 10.0 \\
Concha (N ${ }^{\circ}$ de espiras) & 1.5 & 0 & 100.0 & 0 & 100.0 & 0 & 100.0 & 0 & 100.0 \\
& 2.0 & 2 & 100.0 & 2 & 36.7 & 2 & 100.0 & 2 & 100.0 \\
& 3.0 & 13 & 6.7 & 13 & 40.0 & 6 & 20.0 & 6 & 10.0 \\
& 4.0 & 30 & 3.3 & 30 & 3.3 & 28 & 20.0 & 28 & 10.0 \\
Corazón funcional & 4.5 & 37 & 33.3 & - & - & 33 & 26.7 & 36 & 6.7 \\
Opérculo & Larval + adulto & 2 & 40.0 & 3 & 56.7 & 2 & 100.0 & 2 & 100.0 \\
& Elongación & 13 & 10.0 & 14 & 13.3 & 15 & 36.7 & 11 & 10.0 \\
Branquias & Forma final & 33 & 13.3 & 30 & 23.3 & 28 & 20.0 & 28 & 13.3 \\
Conducta nado-reptante & Presencia & 39 & 53.3 & 36 & 23.3 & 34 & 30.0 & 37 & 23.3 \\
\hline
\end{tabular}

- : Característica no desarrollada. Control $=28{ }^{\circ} \mathrm{C}-\mathrm{pH} 8.1, \mathrm{~T} 1=28{ }^{\circ} \mathrm{C}-\mathrm{pH} 7.6, \mathrm{~T} 2=31^{\circ} \mathrm{C}-\mathrm{pH} 8.1, \mathrm{~T} 3=31^{\circ} \mathrm{C}-\mathrm{pH} 7.6$. - : Feature not developed. Control $=28{ }^{\circ} \mathrm{C}-\mathrm{pH} 8.1, \mathrm{~T} 1=28{ }^{\circ} \mathrm{C}-\mathrm{pH} 7.6, \mathrm{~T} 2=31^{\circ} \mathrm{C}-\mathrm{pH} 8.1, \mathrm{~T} 3=31{ }^{\circ} \mathrm{C}-\mathrm{pH} 7.6$.

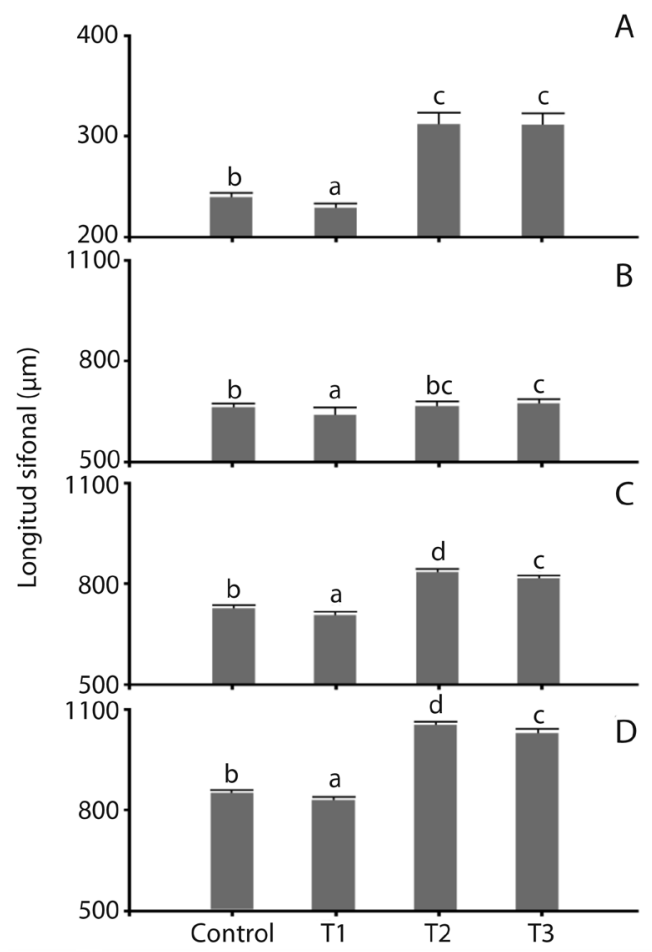

(Fig. 5). No se presentó variación significativa para la proporción de Calcio entre tratamientos $\left(\mathrm{H}_{(\mathrm{g} .1 .3)}=0.57, \mathrm{P}_{(\alpha 0.05)}=0.9038\right)$.

Raman: Se detectaron tres picos para aragonita $\left(206,705\right.$ y $\left.1085 \mathrm{~cm}^{-1}\right)$ y Calcita $(282$, 711 y $1085 \mathrm{~cm}^{-1}$ ) (Fig. 6). El pico de 1085 $\mathrm{cm}^{-1}$ mostró mayor intensidad en $31{ }^{\circ} \mathrm{C}(\mathrm{pH}$ $7.6=3338.77 \pm 1046.86$ a.u. y $\mathrm{pH} 8.1=1$ $259.15 \pm 608.35$ a.u.). El segundo pico (de 705 a $711 \mathrm{~cm}^{-1}$ ) registró más intensidad en $\mathrm{pH} 7.6$

Fig. 3. Crecimiento en larvas de $S$. gigas para los tratamientos de temperatura $\left({ }^{\circ} \mathrm{C}\right)$ y $\mathrm{pH}$. Edades $=\mathrm{A}: 0$ días $/$ Eclosión, B: 10 días, C: 20 días, y D: 30 días; Control = $28^{\circ} \mathrm{C}-\mathrm{pH} 8.1, \mathrm{~T} 1=28{ }^{\circ} \mathrm{C}-\mathrm{pH} 7.6, \mathrm{~T} 2=31^{\circ} \mathrm{C}-\mathrm{pH} 8.1$, $\mathrm{T} 3=31{ }^{\circ} \mathrm{C}-\mathrm{pH}$ 7.6. a-d: grupos generados a partir de la comparación de medias de Kruskal-Wallis ( $\alpha$ 0.05).

Fig. 3. Growth of the $S$. gigas larvae for temperature $\left({ }^{\circ} \mathrm{C}\right)$ and $\mathrm{pH}$ treatments. Larval age $=\mathrm{A}: 0$ days-old (d.o.)/ Hatchery, B: 10 d.o., C: 20 d.o., and D: 30 d.o.; Control = $28{ }^{\circ} \mathrm{C}-\mathrm{pH} 8.1, \mathrm{~T} 1=28{ }^{\circ} \mathrm{C}-\mathrm{pH} 7.6, \mathrm{~T} 2=31{ }^{\circ} \mathrm{C}-\mathrm{pH} 8.1$, $\mathrm{T} 3=31{ }^{\circ} \mathrm{C}-\mathrm{pH}$ 7.6. a-d: groups obtained from average comparisons by Kruskal-Wallis analysis $(\alpha 0.05)$. 


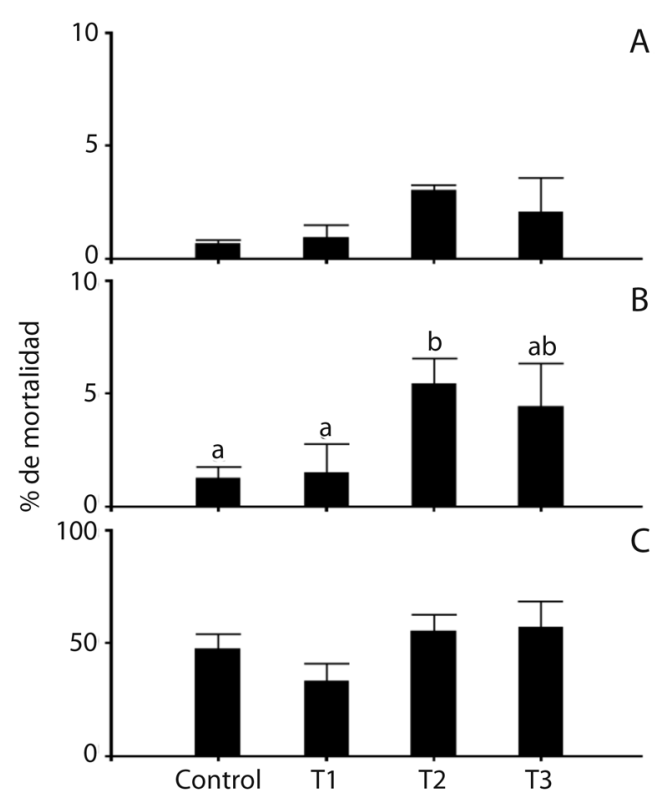

Fig. 4. Porcentaje de mortalidad para larvas de S. gigas, determinado durante los recambios de agua para los tratamientos de temperatura y $\mathrm{pH}$. Edades $=\mathrm{A}: 10$ días, B: 20 días, y C: 30 días; Control $=28^{\circ} \mathrm{C}-\mathrm{pH} 8.1, \mathrm{~T} 1=28$ ${ }^{\circ} \mathrm{C}-\mathrm{pH} 7.6, \mathrm{~T} 2=31{ }^{\circ} \mathrm{C}-\mathrm{pH} 8.1, \mathrm{~T} 3=31{ }^{\circ} \mathrm{C}-\mathrm{pH}$ 7.6. a-d: grupos generados a partir de la comparación de medias de Kruskal-Wallis $(\alpha 0.05)$.

Fig. 4. Percentage of mortality for $S$. gigas larvae, calculated from the water changes for the temperature and $\mathrm{pH}$ treatments. Larval age $=\mathrm{A}: 10$ days-old (d.o.), B: 20 d.o., and C: 30 d.o.; Control $=28{ }^{\circ} \mathrm{C}-\mathrm{pH} 8.1, \mathrm{~T} 1=28{ }^{\circ} \mathrm{C}$ - pH 7.6, T2 $=31{ }^{\circ} \mathrm{C}-\mathrm{pH} 8.1, \mathrm{~T} 3=31{ }^{\circ} \mathrm{C}-\mathrm{pH}$ 7.6. a-d: groups obtained from average comparisons by KruskalWallis analysis $(\alpha 0.05)$.

$\left(28^{\circ} \mathrm{C}=259.15 \pm 121.02\right.$ a.u. y $31^{\circ} \mathrm{C}=606.50$ \pm 111.80 a.u.). Se detectó aragonita en todos los tratamientos, con mayor ocurrencia en $28{ }^{\circ} \mathrm{C}$ (100\%, independientemente del pH). La intensidad de aragonita $\left(206 \mathrm{~cm}^{-1}\right)$ fue mayor en $\mathrm{pH}$ 8.1 (Control: $1039.54 \pm 780.26$ a.u. y $31{ }^{\circ} \mathrm{C}$ : $1228.87 \pm 0.00$ a.u.) (Fig. 7). Se registró calcita $\left(282 \mathrm{~cm}^{-1}\right)$ solo en larvas cultivadas a $31{ }^{\circ} \mathrm{C}$ (pH $8.1=80 \%$; pH $7.6=17 \%$ ), la intensidad de este carbonato fue mayor en $\mathrm{pH} 8.1$ (206.04 \pm 122.33 a.u.) (Fig. 7).

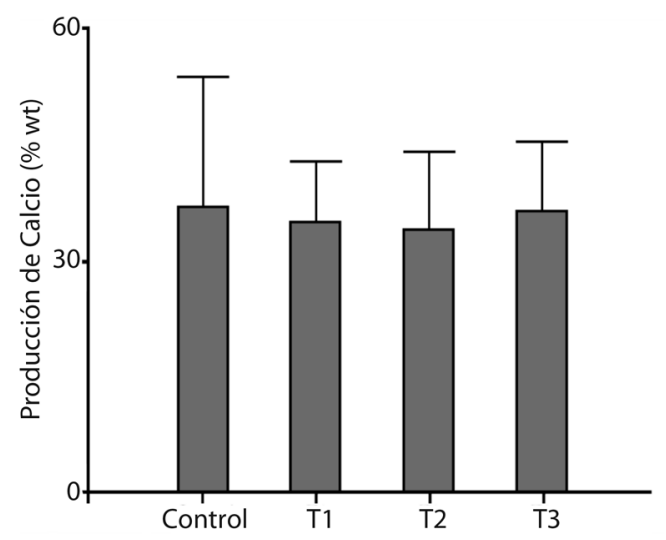

Fig. 5. Calcificación media de la concha en larvas de $S$. gigas (edad $=30$ días). Análisis de Espectroscopia de Energía Dispersiva (EDX). \% $w t=$ porcentaje en peso; $\mathrm{n}$ $=3$ larvas por tratamiento. Control $=28^{\circ} \mathrm{C}-\mathrm{pH} 8.1, \mathrm{~T} 1=$ $28{ }^{\circ} \mathrm{C}-\mathrm{pH} 7.6, \mathrm{~T} 2=31{ }^{\circ} \mathrm{C}-\mathrm{pH} 8.1, \mathrm{~T} 3=31{ }^{\circ} \mathrm{C}-\mathrm{pH}$ 7.6. Fig. 5. Average calcification of $S$. gigas larval shell (30 days old) obtained by Energy-Dispersive X-ray Analysis $(\mathrm{EDX}) . \% w t=$ percent in weight; $\mathrm{n}=3$ larvae per treatment. Control $=28{ }^{\circ} \mathrm{C}-\mathrm{pH} 8.1, \mathrm{~T} 1=28^{\circ} \mathrm{C}-\mathrm{pH} 7.6$, $\mathrm{T} 2=31{ }^{\circ} \mathrm{C}-\mathrm{pH} 8.1, \mathrm{~T} 3=31{ }^{\circ} \mathrm{C}-\mathrm{pH} 7.6$.

\section{DISCUSIÓN}

El calentamiento y acidificación del océano podrían tener efectos severos en organismos calcificadores (Gazeau et al., 2007). Las larvas de organismos marinos, al ser más vulnerables a cambios ambientales, son empleadas como indicador del efecto del incremento de la temperatura y reducción del $\mathrm{pH}$ a través del estudio de las tasas de eclosión, desarrollo, crecimiento, mortalidad y calcificación en larvas de invertebrados (Sale et al., 2010; Talmage \& Gobler, 2009; Ushakova, 2003).

En general, los estudios de cambio climático se han desarrollado con especies de ambientes fríos a templados dentro de las cuales destacan Haliotis sp. y Mytilus sp. (Gaylord et al., 2011; Gazeau et al., 2010, 2011; Miller, Reynolds, Sobrino, \& Riedel, 2009; Sunday, Crim, Harley, \& Hart, 2011), siendo escasas las investigaciones con moluscos de ambientes tropicales. 


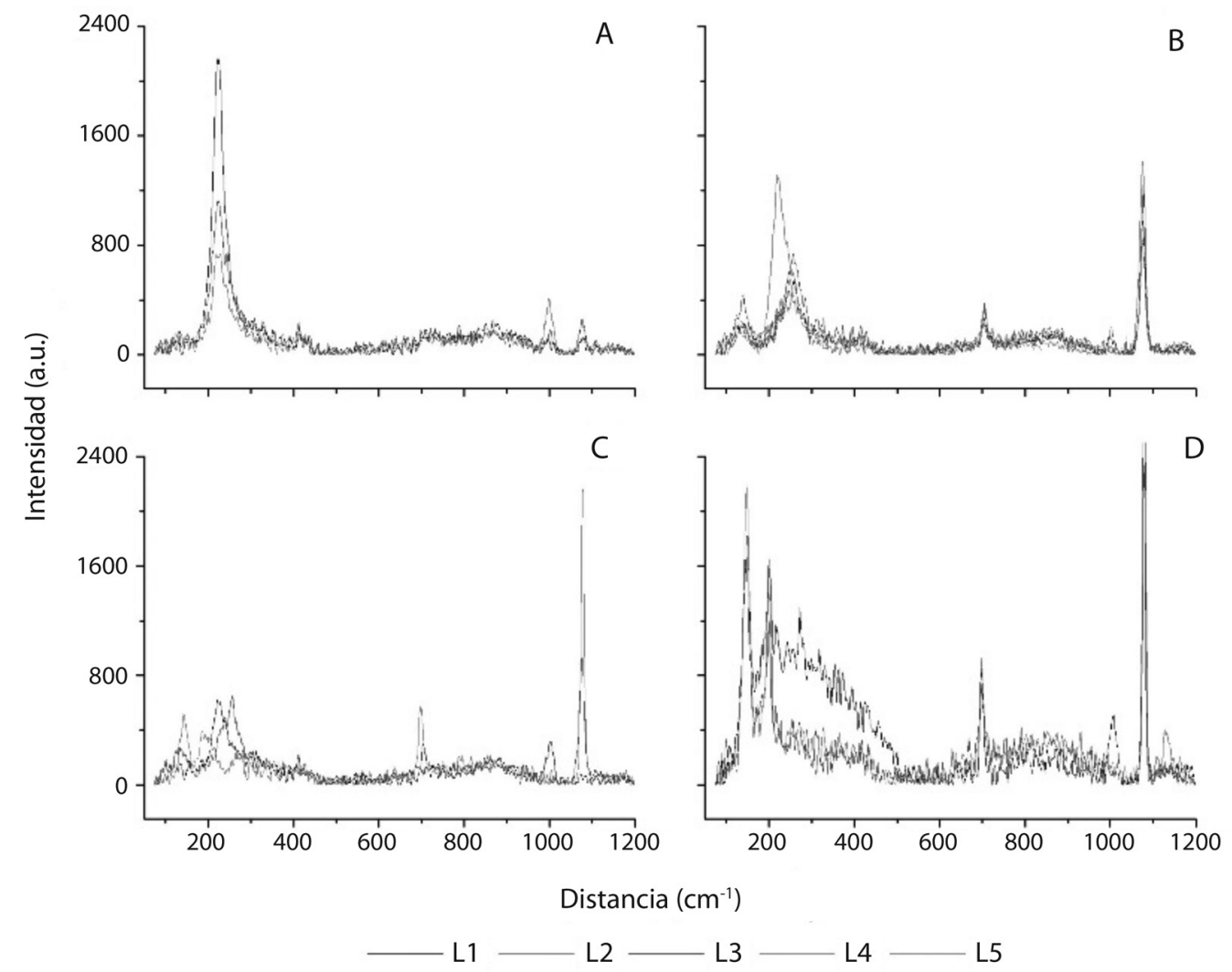

Fig. 6. Calcificación de la concha en larvas de $S$. gigas (edad = 30 días). Análisis RAMAN, $\mathrm{n}=3-5$ larvas por tratamiento. A: $28{ }^{\circ} \mathrm{C}-\mathrm{pH} 8.1$ (Control); B: $31{ }^{\circ} \mathrm{C}-\mathrm{pH} 8.1$; C: $28{ }^{\circ} \mathrm{C}-\mathrm{pH} 7.6$; y D: $31{ }^{\circ} \mathrm{C}-\mathrm{pH} 7.6$; a.u.: unidades arbitrarias; L 1-5: Larvas 1-5.

Fig. 6. Calcification in S. gigas larval shell (30 days old) measured by RAMAN Spectroscopy, $\mathrm{n}=3-5$ larvae per treatment. A: $28{ }^{\circ} \mathrm{C}-\mathrm{pH} 8.1$ (Control); B: $31{ }^{\circ} \mathrm{C}-\mathrm{pH} 8.1$; $\mathrm{C}: 28^{\circ} \mathrm{C}$ - $\mathrm{pH} 7.6$; y D: $31{ }^{\circ} \mathrm{C}$ - $\mathrm{pH}$ 7.6; a.u.: arbitrary units; L 1-5: Larvae 1-5.

Al evaluar la tasa de eclosión Parker, Ross, y O'Connor (2010), en tratamientos combinados de temperatura $\left(18,22,26\right.$ y $\left.30^{\circ} \mathrm{C}\right) \mathrm{y} \mathrm{pH}$ $(8.2,8.0,7.9$ y 7.8$)$, para Saccostrea glomerata y $C$. gigas, obtuvieron $100 \%$ de larva $\mathrm{D}$ en condiciones normales $\left(26{ }^{\circ} \mathrm{C}-\mathrm{pH} 8.2\right)$; sin embargo, observaron menor proporción de larvas al disminuir la temperatura y el $\mathrm{pH}$ (18 ${ }^{\circ} \mathrm{C}-\mathrm{pH}$ 7.8: C. gigas $=35 \%$ y S. glomerata $=58 \%$ ). Por otra parte, Gazeau et al. (2011) reportan menor porcentaje de eclosión para embriones de $C$. gigas al disminuir el $\mathrm{pH}$ de 8.03 a 7.41 (de 95 a $20 \%$ ). En el caso de $S$. gigas, la tasa de eclosión no se vio influenciada por ningún parámetro, sin embargo, se puede determinar que el tiempo de incubación se reduce, al incrementar la temperatura, independientemente del $\mathrm{pH}$.

Aldana, Lucas, Brulé, Salguero, y Rendon (1989) reportan el crecimiento para Strombus costatus en función de la temperatura, y registraron tallas de $812 \mu \mathrm{m}$ en $28{ }^{\circ} \mathrm{C}$ y $685 \mu \mathrm{m}$ en $24{ }^{\circ} \mathrm{C}$ (para larvas de 31 días). En cuanto a la acidificación, se reportaron menores tasas de crecimiento para larvas de Mercenaria mercenaria, Argopecten irradians y $C$. virginica cultivadas en pH 7.5 (Talmage \& Gobler, 2009), en el presente estudio observamos que si la temperatura se mantiene constante $\left(28^{\circ} \mathrm{C}\right)$ y el $\mathrm{pH}$ disminuye, el crecimiento de $S$. gigas 


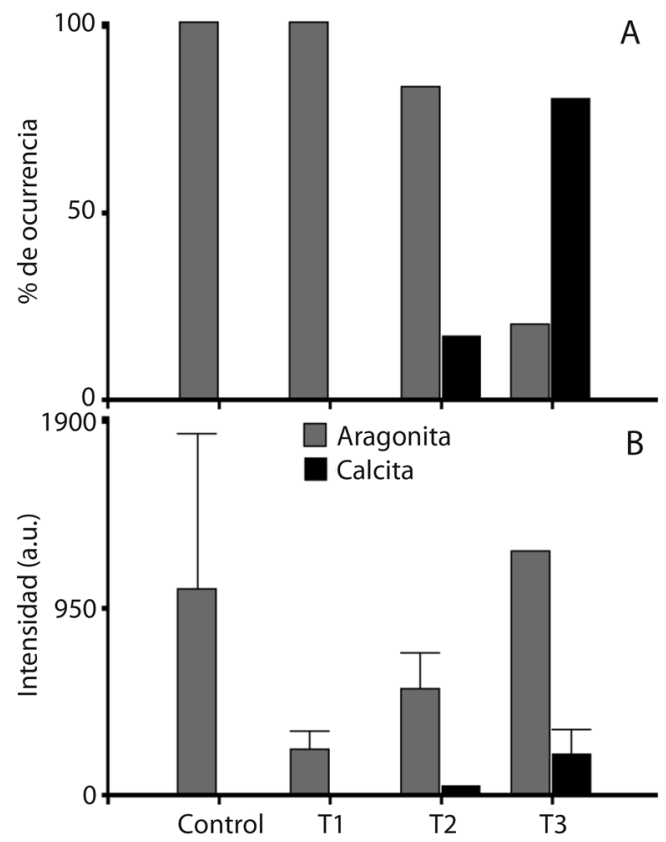

Fig. 7. Proporción (A) e intensidad (B) de los carbonatos aragonita y calcita obtenidos por análisis RAMAN, en las conchas de larvas de $S$. gigas de 30 días. Larvas analizadas por tratamiento $=3-5$. Control $=28{ }^{\circ} \mathrm{C}-\mathrm{pH} 8.1, \mathrm{~T} 1=28$ ${ }^{\circ} \mathrm{C}-\mathrm{pH} 7.6, \mathrm{~T} 2=31^{\circ} \mathrm{C}-\mathrm{pH} 8.1, \mathrm{~T} 3=31^{\circ} \mathrm{C}-\mathrm{pH}$ 7.6; a.u.: unidades arbitrarias.

Fig. 7. Proportions (A) and intensity (B) of the carbonates aragonite and calcite, measured by RAMAN Spectroscopy in the $S$. gigas larval shell of 30 days old. Larvae per treatment $=3-5$. Control $=28{ }^{\circ} \mathrm{C}-\mathrm{pH} 8.1, \mathrm{~T} 1=28{ }^{\circ} \mathrm{C}$ - $\mathrm{pH} 7.6, \mathrm{~T} 2=31{ }^{\circ} \mathrm{C}-\mathrm{pH} 8.1, \mathrm{~T} 3=31{ }^{\circ} \mathrm{C}-\mathrm{pH}$ 7.6; a.u.: arbitrary units.

es menor, en contraparte, al aumentar la temperatura, el efecto del $\mathrm{pH}$ es contrarrestado, los que beneficia el crecimiento del organismo.

Se conoce que el incremento de la temperatura y la disminución del $\mathrm{pH}$ tienen un efecto negativo en las tasas de mortalidad larval de moluscos. Aldana Aranda et al. (1989) registraron mortalidades de 83 a $90 \%$ para $S$. costatus ante incrementos de temperatura (de 24 a $28{ }^{\circ} \mathrm{C}$, respectivamente). En cuanto a la acidificación, Talmage y Glober $(2009,2010)$ observaron que a menor $\mathrm{pH}$ (7.5) la mortalidad diaria incrementa para $M$. mercenaria (de 1.8 a $6.7 \%$ ) y $A$. irradians, (de 3.8 a $7.8 \%$ ). Resultados similares fueron presentados por Kurihara,
Kato, e Ishimatsu (2007) y Gazeau et al. (2011) para C. gigas, donde la mortalidad aumentó de 20 a $90 \%$ al reducirse el pH (de 8.1 a 7.6, respectivamente). Los resultados de este estudio mostraron un mayor porcentaje de mortalidad hacia el día 20 de desarrollo, observando que las larvas cultivadas a $31{ }^{\circ} \mathrm{C}$ fueron más afectadas a nivel fisiológico por la temperatura.

La acidificación del medio marino produce baja saturación de aragonita y calcita, lo cual afecta directamente a organismos calcificadores al reducirse la tasa de incorporación de calcio en sus concha (Talmage \& Gobler, 2009). Kurihara et al. (2007) y Gazeau et al. (2011), señalaron que la tasa de calcificación en larvas de $C$. gigas disminuye de 72 a $30 \%$ ante menor $\mathrm{pH}$ (de 8.1 a 7.4, respectivamente); contrariamente, en este estudio se observó que la proporción de calcio en el estadio planctónico de $S$. gigas, no mostró variación en ninguna condición experimental.

Weiss, Tuross, Addadi, y Weiner (2002) informaron que en estadios iniciales, la concha de $M$. mercenaria y $C$. gigas se compone de carbonato de calcio amorfo, sustituyéndose por aragonita al final de la vida larvaria. Auzoux-Bordenave et al. (2010) presentaron una revisión para siete especies de moluscos, entre ellas Ostrea edulis y Pinctada fucata, en la que señalaron que la protoconcha se compone por aragonita y trazas de carbonato amorfo, mientras que la teleoconcha por calcita. En este estudio, las conchas de las larvas de 30 días (premetamórficas) de todos los tratamientos, presentaron aragonita, mientras que calcita solo se observó en organismos cultivados a $31{ }^{\circ} \mathrm{C}$. Ambos polimorfos de carbonato de calcio mostraron menores registros en función del $\mathrm{pH}$.

Se sabe que el incremento de temperatura puede aumentar los efectos negativos de la acidificación del océano, cuando pasan el umbral de tolerancia térmica, ya que puede alterar la membrana celular y modificar la homeóstasis ácido-base; también puede influir en la actividad de las proteínas implicadas en la regulación ácido-base (O’Donnell, Hammond, \& Hofmann, 2008; Padilla-Gamiño, Kelly, Evans, \& Hofmann, 2013; Pörtner, 2010). De acuerdo 
con Le Moullac et al. (2016), las larvas pueden adaptarse a condiciones de acidificación, siendo la temperatura el principal estresor en el desarrollo larvario. En este estudio se observó que $S$. gigas podría adaptarse al calentamiento y acidificación de los océanos, sin embargo, los procesos de biomineralización de la concha fueron afectados, razón por la cual las conchas de $S$. gigas mostraron mayor proporción de aragonita (carbonato presente en las fases tempranas del desarrollo larvario), retardándose así el proceso de formación de calcita. Aunque la aragonita es el polimorfo de carbonato de calcio menos estable, su presencia en todos los tratamientos podría atribuirse a la dureza de este carbonato, por lo cual, se detectó aún en condiciones de $\mathrm{pH}$ menor, no obstante con menores intensidades, lo cual, se traduce en la formación de conchas con menor densidad o estructuras menos compactas, lo que podría generar repercusiones a nivel fisiológico.

$\mathrm{Si}$ bien, se determinaron las fases (presencia/ausencia) e intensidades de aragonita y calcita de la concha de $S$. gigas, se debe considerar realizar un análisis químico a fin de conocer la variación o estratificación de estos dos carbonatos de calcio, a fin de conocer a fondo los efectos de la acidificación, empleando diferentes ambientes de $\mathrm{pH}$, a través de la vida larvaria de $S$. gigas.

\section{AGRADECIMIENTOS}

Agradecemos al Consejo Nacional de Ciencia y Tecnología por financiar el presente estudio (Proyecto: "El caracol rosa como indicador de Cambio climático en el Caribe: Calentamiento y Acidificación Oceánica: CB-2011-01/181329). Al Centro de Investigación y de Estudios Avanzados del IPN, Unidad Mérida, en especial a Ana Ruth Cristóbal Ramos y José Bante Guerra por su apoyo técnico en la obtención de datos mediante EDX y Microscopía RAMAN. A Geonel Rodríguez Gattorno por su contribución en el análisis RAMAN.

\section{RESUMEN}

El aumento de las emisiones de $\mathrm{CO}_{2}$ produce calentamiento y reducción del $\mathrm{pH}$ en los océanos, lo cual puede generar efectos negativos en muchos organismos marinos, particularmente en aquellos con estructuras calcáreas (i.e. moluscos), afectando principalmente a sus estadios larvarios (Le Moullac et al., 2016), en este sentido, se estudió a $S$. gigas, gasterópodo de importancia comercial en el mar Caribe, con el fin de conocer el efecto de la temperatura y la acidificación en el desarrollo, el crecimiento, la mortalidad y la calcificación durante su fase larvaria. Se realizó un cultivo larvario empleando cuatro tratamientos de temperatura y $\mathrm{pH}\left(\right.$ control $=28{ }^{\circ} \mathrm{C}-\mathrm{pH} 8.1, \mathrm{~T} 1=28$ ${ }^{\circ} \mathrm{C} \mathrm{pH} \mathrm{7.6,} \mathrm{T2}=31{ }^{\circ} \mathrm{C} \mathrm{pH} 8.1$ y T3 $=31{ }^{\circ} \mathrm{C}-\mathrm{pH}$ 7.6) por triplicado. La eclosión se registró al inicio del experimento (No. de huevos - No. de larvas nacidas), por otra parte, el desarrollo de órganos, el crecimiento de la concha y la mortalidad se evaluaron a través del tiempo. La calcificación fue estudiada mediante análisis EDX y Raman para larvas de 30 días de edad. Se observó que el desarrollo y el crecimiento de órganos fue mayor a $31{ }^{\circ} \mathrm{C}$ (talla inicial $=230$ \pm 4.12 a $313.27 \pm 11.34 \mu \mathrm{m}$, talla final $=829.50 \pm 11.33$ a $1054.50 \pm 11.13 \mu \mathrm{m}$; para $\mathrm{T} 1$ y $\mathrm{T} 2$, respectivamente), mismo patrón se presentó para el tiempo de eclosión (18 hr) y la tasa de mortalidad ( $57 \%$ ). La proporción de calcio $(\% w t)$ fue similar entre tratamientos (de $34.37 \pm 10.05$ a $37.29 \pm 16.81 \% w t)$. El análisis Raman mostró aragonita para las conchas de todas las condiciones experimentales, con valores más altos en el control (1 $039.54 \pm 780.26$ a.u.). Calcita solo se detectó en los tratamientos de $31^{\circ} \mathrm{C}(174.56$ \pm 127.19 a.u.), mientras que, a menor $\mathrm{pH}$ (7.6), la intensidad de aragonita y calcita fue menor. En conclusión, $S$. gigas podría adaptarse a escenarios futuros de temperatura y acidificación, sin embargo, puede verse afectado durante los procesos de biomineralización de la concha.

Palabras clave: desarrollo, temperatura, acidificación, calcificación, Strombus gigas.

\section{REFERENCIAS}

Aldana-Aranda, D., Lucas, A., Brule, T., Salguero, E., \& Rendon, F. (1989). Effects of temperature, algal food, feeding rate and density on the larval growth of the milk conch (Strombus costatus) in Mexico. Aquaculture, 76(3), 361-371. http://doi. org/10.1016/0044-8486(89)90087-2

Auzoux-Bordenave, S., Badou, A., Gaume, B., Berland, S., Helléouet, M. N., Milet, C., \& Huchette, S. (2010). Ultrastructure, chemistry and mineralogy of the growing shell of the European abalone Haliotis tuberculata. Journal of Structural Biology, 171(3), 277-290. http://doi.org/10.1016/j.jsb.2010.05.012

Brito-Manzano, N., Aldana-Aranda, D., de la Cruz-Lázaro, E., \& Estrada-Botello, M. A. (2006). Organogénesis 
larvaria de Strombus gigas (Mesogastropoda: Strombidae) en el Arrecife Alacranes durante el período máximo de su época reproductiva. Revista Universidad y Ciencia, 22(1), 75-82.

Caldeira, K., \& Wickett, M. E. (2003). Oceanography: Anthropogenic carbon and ocean pH. Nature, 425(6956), 365-365. http://doi.org/10.1038/425365

Chollett, I., Mumby, P. J., Müller-Karger, F. E., \& Hu, C. (2012). Physical environments of the Caribbean Sea. Limnology and Oceanography, 57(4), 1233-1244. http://doi.org/10.4319/1o.2012.57.4.1233

Enriquez-Diaz, M. R., Volland, J. M., Chavez-Villegas, J. F., Aldana-Aranda, D., \& Gros, O. (2015). Development of the planktotrophic veligers and plantigrades of Strombus pugilis (Gastropoda). Journal of Molluscan Studies, eyv011. http://doi.org/10.1093/mollus/ eyv011

Gaylord, B., Hill, T. M., Sanford, E., Lenz, E. A., Jacobs, L. A., Sato, K. N., ... Hettinger, A. (2011). Functional impacts of ocean acidification in an ecologically critical foundation species. Journal of Experimental Biology, 214(15), 2586-2594. http://doi.org/10.1242/ jeb.055939

Gazeau, F., Gattuso, J. P., Dawber, C., Pronker, A. E., Peene, F., Peene, J., ... Middelburg, J. J. (2010). Effect of ocean acidification on the early life stages of the blue mussel Mytilus edulis. Biogeosciences, 7(7), 2051-2060. http://doi.org/10.5194/bg-7-2051-2010

Gazeau, F., Gattuso, J. P., Greaves, M., Elderfield, H., Peene, J., Heip, C. H. R., \& Middelburg, J. J. (2011). Effect of Carbonate Chemistry Alteration on the Early Embryonic Development of the Pacific Oyster (Crassostrea gigas). PLOS One, 6(8), e23010. http:// doi.org/10.1371/journal.pone.0023010

Gazeau, F., Quiblier, C., Jansen, J. M., Gattuso, J. P., Middelburg, J. J., \& Heip, C. H. R. (2007). Impact of elevated $\mathrm{CO}_{2}$ on shellfish calcification. Geophysical Research Letters, 34(7), L07603. http://doi. org/10.1029/2006GL028554

Gledhill, D. K., Wanninkhof, R., Millero, F. J., \& Eakin, M. (2008). Ocean acidification of the Greater Caribbean Region 1996-2006. Journal of Geophysical Research: Oceans, 113(C10), C10031. http://doi. org/10.1029/2007JC004629

Intergovernmental Panel on Climate Change. (2007). Impacts, Adaptation and Vulnerability. Contribution of Working Group II to the Fourth Assessment Report of the Intergovernmental Panel on Climate Change. Cambridge University Press. Retrieved from https://www.ipcc.ch/pdf/assessment-report/ar4/wg2/ ar4_wg2_full_report.pdf

Kurihara, H., Kato, S., \& Ishimatsu, A. (2007). Effects of increased seawater $\mathrm{pCO}_{2}$ on early development of the oyster Crassostrea gigas. Aquatic Biology, 1(1), 91-98. http://doi.org/10.3354/ab00009

Le Moullac, G., Soyez, C., Latchere, O., Vidal-Dupiol, J., Fremery, J., Saulnier, D., ... Gueguen, Y. (2016). Pinctada margaritifera responses to temperature and $\mathrm{pH}$ : Acclimation capabilities and physiological limits. Estuarine, Coastal and Shelf Science. http:// doi.org/10.1016/j.ecss.2016.04.011

Magaña Rueda, V., Graizbord, B., Buenfil Friedman, J., \& Gómez Mendoza, L. (2009). Escenarios de cambio climático y tendencias en la zona del Golfo de México. In J. Buenfil (Ed.). Adaptación a los impactos del cambio climático en los humedales costeros del Golfo de México (Vol. 2, pp. 569-673). México D.F.: Secretaría de Medio Ambiente y Recursos Naturales / Instituto Nacional de Ecología (INE-SEMARNAT). Retrieved from http://www2.inecc.gob.mx/publicaciones/libros/610/cap6.pdf

Miller, A. W., Reynolds, A. C., Sobrino, C., \& Riedel, G. F. (2009). Shellfish Face Uncertain Future in High $\mathrm{CO}_{2}$ World: Influence of Acidification on Oyster Larvae Calcification and Growth in Estuaries. PLOS One, 4(5), e5661. http://doi.org/10.1371/journal. pone. 0005661

Nehrke, G., Poigner, H., Wilhelms-Dick, D., Brey, T., \& Abele, D. (2012). Coexistence of three calcium carbonate polymorphs in the shell of the Antarctic clam Laternula elliptica. Geochemistry, Geophysics, Geosystems - Wiley Online Library, 13(5), Q05014. http://doi.org/10.1029/2011GC003996

O’Donnell, M. J., Hammond, L. M., \& Hofmann, G. E. (2008). Predicted impact of ocean acidification on a marine invertebrate: elevated $\mathrm{CO}_{2}$ alters response to thermal stress in sea urchin larvae. Marine Biology, 156(3), 439-446. http://doi.org/10.1007/ s00227-008-1097-6

Orr, J. C., Fabry, V. J., Aumont, O., Bopp, L., Doney, S. C., Feely, R. A., ... Yool, A. (2005). Anthropogenic ocean acidification over the twenty-first century and its impact on calcifying organisms. Nature, 437(7059), 681-686. http://doi.org/10.1038/nature04095

Padilla-Gamiño, J. L., Kelly, M. W., Evans, T. G., \& Hofmann, G. E. (2013). Temperature and $\mathrm{CO}_{2}$ additively regulate physiology, morphology and genomic responses of larval sea urchins, Strongylocentrotus purpuratus. Proceedings of the Royal Society of London B: Biological Sciences, 280(1759), 20130155. http:// doi.org/10.1098/rspb.2013.0155

Parker, L. M., Ross, P. M., \& O’Connor, W. A. (2010). Comparing the effect of elevated $\mathrm{pCO}_{2}$ and temperature on the fertilization and early development of two species of oysters. Marine Biology, 157(11), 24352452. http://doi.org/10.1007/s00227-010-1508-3

Pörtner, H. O. (2010). Oxygen- and capacity-limitation of thermal tolerance: a matrix for integrating 
climate-related stressor effects in marine ecosystems. Journal of Experimental Biology, 213(6), 881-893. http://doi.org/10.1242/jeb.037523

Roller, R. A., \& Stickle, W. B. (1989). Temperature and salinity effects on the intracapsular development, metabolic rates, and survival to hatching of Thais haemastoma canaliculata (Gray) (Prosobranchia:Muricidae) under laboratory conditions. Journal of Experimental Marine Biology and Ecology, 125(3), 235-251. http://doi. org/10.1016/0022-0981(89)90099-3

Sale, P. F., Van Lavieren, H., Ablan, M. C., Atema, J., Butler, M., Fauvelot, C., ... \& Stewart, H. L. (2010). Conservando la Conectividad de los Arrecifes: Guía Para los Administradores de las Áreas Marinas Protegidas. Grupo de Trabajo de Conectividad, Programa de Investigación Dirigido a los Arrecifes de Coral y a la Creación de Capacidades para la Gestión, UNU-INWEH. Retrieved from http://ccres.net/images/uploads/publications/2/crtr_connectivityhandbook_web_spanish.pdf

Sunday, J. M., Crim, R. N., Harley, C. D. G., \& Hart, M. W. (2011). Quantifying rates of evolutionary adaptation in response to ocean acidification. PloS One, 6(8), e22881. http://doi.org/10.1371/journal.pone.0022881

Talmage, S. C., \& Gobler, C. J. (2009). The effects of elevated carbon dioxide concentrations on the metamorphosis, size, and survival of larval hard clams
(Mercenaria mercenaria), bay scallops (Argopecten irradians), and Eastern oysters (Crassostrea virginica). Limnology and Oceanography, 54(6), 20722080. http://doi.org/10.4319/lo.2009.54.6.2072

Talmage, S. C., \& Gobler, C. J. (2010). Effects of past, present, and future ocean carbon dioxide concentrations on the growth and survival of larval shellfish. Proceedings of the National Academy of Sciences of the United States of America, 107(40), 17246-17251. http://doi.org/10.1073/pnas.0913804107

Ushakova, O. O. (2003). Combined effect of salinity and temperature on Spirorbis spirorbis L. and Circeus spirillum L. larvae from the White Sea. Journal of Experimental Marine Biology and Ecology, 296(1), 23-33.

Watson, S. A., Southgate, P. C., Tyler, P. A., \& Peck, L. S. (2009). Early Larval Development of the Sydney Rock Oyster Saccostrea glomerata Under Near-Future Predictions of $\mathrm{CO}_{2}$-Driven Ocean Acidification. Journal of Shellfish Research, 28(3), 431-437. http:// doi.org/10.2983/035.028.0302

Weiss, I. M., Tuross, N., Addadi, L., \& Weiner, S. (2002). Mollusc larval shell formation: amorphous calcium carbonate is a precursor phase for aragonite. Journal of Experimental Zoology, 293(5), 478-491. http://doi. org/10.1002/jez.90004 
